\title{
Finite nucleus effects, relativistic effects and picture change error in the IOTC/DKH2 contact spin densities of $\mathrm{Cu}, \mathrm{Ag}$, Au atoms
}

\author{
Michal Malček, Lukáš Bučinský, Stanislav Biskupič \\ Institute of Physical Chemistry and Chemical Physics, Faculty of Chemical and Food Technology, \\ Slovak University of Technology in Bratislava, Radlinského 9, SK-812 37 Bratislava, Slovak Republic \\ michal.malcek@stuba.sk
}

\begin{abstract}
Sensitivity of contact spin density as well as electron density to the size of nucleus is investigated using the Gaussian model of nucleus and the point charge nucleus model. Scalar Infinite Order Two Component and scalar second order Douglas-Kroll-Hess quasirelativistic contact spin densities (spin densities at the nucleus) of $\mathrm{Cu}, \mathrm{Ag}$ and $\mathrm{Au}$ atoms are considered. The non-relativistic contact spin densities and the valence $s$-orbital contact densities of Kramers restricted orbitals (Cu, Ag, Au atoms) are presented as well. The picture change error in the quasirelativistic calculations of spin densities is corrected by analytic means. Uncontracted triple-zeta $\mathrm{UTZ}+\mathrm{N} s$ basis sets are employed, where $\mathrm{N}$ is the number of additional tight $s$ Gaussians. In addition, the impact of tight $p$ and $d$ Gaussians is briefly discussed.
\end{abstract}

Keywords: contact spin density, nucleus model, PCE correction, picture change error

\section{Introduction}

The last two decades were in the sign of a rapid development of relativistic methods in computational chemistry [Pyykkö 1988, Schwerdtfeger (Ed.) 2002, Dyall and Faegri 2007, Reiher and Wolf 2009]. In this paper, $2^{\text {nd }}$ order Douglas-Kroll-Hess (DKH2) [Foldy and Wouthuysen 1950, Douglas and Kroll 1974, Hess 1985, Nakajima and Hirao 2000, Wolf et al. 2002, Reiher and Wolf 2004a, Reiher and Wolf 2004b] and Infinite-Order Two-Component (IOTC) [Barysz et al. 1997, Barysz and Sadlej 2001, Barysz and Sadlej 2002, Iliaš and Saue 2007, Kedziera and Barysz 2007] quasirelativistic approaches, based on the diagonalization of $\hat{H}_{D}$, are employed.

Relativistic effects play a non negligible role in systems containing heavy atoms [Pyykkö 1988, Schwerdtfeger (Ed.) 2002, Dyall and Faegri 2007, Reiher and Wolf 2009, Iliaš et al. 2010]. Foremostly, electron density at the nucleus and properties directly related to core electrons are mostly sensitive to the relativistic effects. In the case of contact electron density (electron density at the nucleus), relativistic effects, picture change error (PCE), sensitivity to the size of the nucleus and the basis set quality at the nucleus have been already studied for a number of atoms [Mastalerz et al. 2010, Bučinský et al. 2011, Peng and Reiher 2011, Malček et al. 2013] and/or simple molecules [Mastalerz et al. 2008, Knecht et al. 2011]. The finite size nucleus model (FN) has to be included [Mastalerz et al. 2008, Mastalerz et al. 2010, Bučinský et al. 2011, Knecht et al. 2011, Malček et al. 2013] because relativistic contact electron density has a weak singularity at the point charge (PCH) nucleus [Kutzelnigg 2004, Mastalerz et al. 2010, Bučinský et al. 2011], i.e. the relativistic wave function is square integrable but infinite at $\mathrm{PCH}$ nucleus. This weak singularity is in the case of finite basis set approach (LCAO using Gaussian functions centered by atoms) coupled with the socalled basis set artifact [Mastalerz et al. 2008], i.e. the LCAO based electron density cannot have a weak singularity contrary to an analytic or numeric [Dyall et al. 1989] solution of the Dirac version of Schrödinger equation.

In addition, calculations at quasirelativistic DKH and/or IOTC level of theory have to take into account the "picture change" of the wave function (PCE), i.e. the unitary transformation of $\hat{H}_{D}$ affects also the wave function [Barysz and Sadlej 2001, Wolf and Reiher 2006a, Wolf and Reiher 2006b]. The formal mapping between the expectation value of a general property operator $\hat{X}$ (it can be also $\hat{H}_{D}$ itself) in the Dirac picture $(\Psi)$ and the DKH/IOTC picture $(\widetilde{\Psi})$ [Barysz and Sadlej 2001, Bučinský et al. 2010, Bučinský et al. 2011] reads:

$$
\langle\Psi|\hat{X}| \Psi\rangle=\left\langle\Psi\left|U^{+} U \hat{X} U^{+} U\right| \Psi\right\rangle=\left\langle\tilde{\Psi}\left|U \hat{X} U^{+}\right| \tilde{\Psi}\right\rangle
$$

The relation ("change of picture") between the transformed DKH/IOTC spinor $\widetilde{\Psi}$ and the Dirac 4-component spinor $\Psi$ is following:

$$
\tilde{\Psi}=U \Psi
$$

To avoid PCE in the DKH/IOTC calculations of properties the transformation of the property operator can be performed as shown in the Equation 1. This PCE correction is actually performed by means of correcting the matrix representation 
of the property operator [Wolf and Reiher 2006a, Wolf and Reiher 2006b]. An alternative option is to move the DKH/IOTC spinor back to the Dirac picture [Barysz et al. 2009].

In this regard, the proper behavior of the spin densities reflecting the size of nucleus, the basis set quality at the nucleus and the behavior of the basis set artifact has not been analyzed yet in full detail. The spin densities (as well as electron densities) have been already reported for a coordination copper compound and the copper atom at the DKH2 level of theory [Bučinský et al. 2012], including PCE correction, but using only the $\mathrm{PCH}$ model of nucleus and only two tight $s$ Gaussian functions. Besides this, the contact electron and spin densities of coinage metal atoms have been studied quite extensively in [Malček et al. 2013] which accounted for the relativistic effects as well as a comparison of both the FN and PCH nucleus model. Nevertheless, the impact of the tight $s$ Gaussian functions on the values of contact electron and spin densities has been considered less systematically. Herein the contact densities of $\mathrm{Cu}, \mathrm{Ag}$ and $\mathrm{Au}$ are revisited, with the focus on their sensitivity to the inclusion of tight $s$ Gaussian functions with the finite basis set. Besides this, the extent of relativistic effects, PCE as well as effects of the size of the nucleus are presented.

\section{Computational details}

The DKH2 calculations were performed in the standard manner as was introduced by Hess and coworkers [Hess 1985, Wolf et al. 2002, Reiher and Wolf 2004a, Reiher and Wolf 2004b]. The calculations at the IOTC level of theory were based on the approach of Barysz and Sadlej [Barysz et al. 1997, Barysz and Sadlej 2001, Barysz and Sadlej 2002, Kedziera and Barysz 2007] and the IOTC $Y$ matrix was obtained by iterative means [Barysz and Sadlej 2002]. All calculations were performed at the scalar (1-component) level of theory.

The $\mathrm{Cu}, \mathrm{Ag}, \mathrm{Au}$ atoms (with spin multiplicity 2 ) were calculated in the electron configuration $[\mathrm{RG}](\mathrm{N}-1)$ $\mathrm{d}^{10} \mathrm{Ns}^{1}$, where $\mathrm{RG}$ means the electron configuration of the closest previous rare gas. Actually, in the case of copper the $[\mathrm{Ar}] 3 \mathrm{~d}^{9} 4 \mathrm{~s}^{2}$ electron configuration is energetically more preferred than $[\mathrm{Ar}] 3 \mathrm{~d}^{10} 4 \mathrm{~s}^{1}$ [Visscher and Dyall 1997]. Nevertheless, in this paper only the $[\mathrm{Ar}] 3 \mathrm{~d}^{10} 4 \mathrm{~s}^{1}$ electron configuration of copper is considered.

The 1-component UHF DKH/IOTC and nonrelativistic calculations were obtained with the TONTO package [Jayatilaka and Grimwood 2000]. The DCH (Dirac-Coulomb Hamiltonian) calculations were performed in Dirac10 [Saue et al. 2010] and Grasp90 [Dyall et al. 1989] packages. The parameters of the Gaussian nucleus model were taken from Visscher and Dyall [Visscher and Dyall 1997]. The uncontracted triple-zeta (UTZ) basis sets of Peterson et al. [Balabanov and Peterson 2005, Peterson and Puzzarini 2005] were employed at the finite basis set level of theory. For more accurate description of contact densities it is found necessary to expand the original basis sets with additional tight s-type Gaussian functions [Mastalerz et al. 2008, Bučinský et al. 2011, Knecht et al. 2011]. Besides the effects of extra $s$ Gaussians, also the effect of extra $p$ and $d$ tight Gaussians will be briefly considered. Exponents of these tight $s, p$ and $d$ Gaussian functions were obtained by assuming a geometric (even tempered) series for the two largest exponents of the original UTZ basis sets [Balabanov and Peterson 2005, Peterson and Puzzarini 2005].

In the case of contact spin density, the following operator has been corrected for PCE:

$$
\hat{\rho}_{s}(\mathbf{r})=\sum_{i=1}^{N} \delta^{(3)}\left(\mathbf{r}_{i}-\mathbf{r}\right) \boldsymbol{\sigma}_{z}
$$

where $\boldsymbol{r}$ is general position, $\boldsymbol{r}_{\mathrm{i}}$ is the position of $i$-th electron, $\delta^{(3)}$ is Dirac delta function and $\sigma_{z}$ is the Pauli $z$ matrix. More details on the analytic PCE correction of electron density can be found in the literature [Mastalerz et al. 2008, Mastalerz et al. 2010, Seino et al. 2010, Bučinský et al. 2010, Knecht et al. 2011]. Actually, the difference between the analytic PCE correction of the spin and electron density is only in the usage of the spin density matrix $\mathbf{D}_{\mathrm{s}}=\mathbf{D}_{\alpha}-\mathbf{D}_{\beta}$ instead of the electron density matrix $\mathbf{D}_{\mathrm{e}}=\mathbf{D}_{\alpha}+\mathbf{D}_{\beta}$ in the evaluation of the density. The PCE correction of the matrix representation of the electron/spin density operator (essentially the $\sigma$ function) remains the same [Bučinský et al. 2011, Bučinský et al. 2012].

The following notation for the spin/electron density will be used throughout the manuscript:

- NR means non-relativistic values

- DKH2 $2_{0}$ employs only $\mathrm{U}_{0}$ - the free particle FoldyWouthuysen (fpFW) transformation within the PGE correction

- DKH2 $2_{1}$ employs $\mathrm{U}_{0} \mathrm{U}_{1}$ - the fpFW and the first order Douglas-Kroll transformations ( D K 1 ) within the PCE correction

- DKH2 employs $\mathrm{U}_{0} \mathrm{U}_{1} \mathrm{U}_{2}$ - the fpFW, DK1 and the second order Douglas-Kroll transformations (DK2) within the PCE correction

- DKH2 $2_{\mathrm{PCE}}$ represents PCE contaminated DKH2 values

- IOTC uses $\mathrm{U}_{0} \mathrm{U}_{1}$ - the fpFW and the IOTC transformations within the PCE correction

- IOTC $_{\mathrm{PCE}}$ represents PCE contaminated IOTC values 


\section{Results \& Discussions}

\section{Cu atom}

The impact of the size of the nucleus (both FN and $\mathrm{PCH}$ ) and of the basis set quality $(\mathrm{UTZ}+\mathrm{N} s)$ on the UHF contact spin densities at the IOTC and NR level of theory are presented in Figures 1a and $1 \mathrm{~b}$. The contact spin/electron densities at NR, DKH2 and IOTC levels of theory (including UTZ and $\mathrm{UTZ}+10 s$ basis sets only and the FN and PCH nucleus models) are compared in the Table 1 and Table 2. Furthermore, the impact of the inclusion of tight $s, p, d$ Gaussians on the contact spin density of copper is shown in Table 3 and the individual orbital contributions are presented in the Table 4 .

Impact of the size of nucleus and basis set quality

The IOTC contact spin density is nearly a linear function of the number of tight $s$ Gaussians which are added to the original UTZ basis set in the case of the PCH model of nucleus, see Figure 1a. This confirms that not only the relativistic electron density [Mastalerz et al. 2008, Knecht et al. 2011, Bučinský et al. 2011] but also the spin density [Kutzelnigg 2004] has a singular behavior at and/ or near to the PCH nucleus. Note that regardless of the nucleus model, the relativistic values of contact spin/electron densities are quite similar in the case of UTZ basis set, while in the case of $\mathrm{UTZ}+10 \mathrm{~s}$ basis set the impact of the nucleus model is obvious (singular behavior of density at nucleus), see Table 1 and Table 2.

In the case of the FN model, the first two added tight $s$ Gaussians have a significant influence on the contact spin density of $\mathrm{Cu}$, while the following $s$ Gaussian functions cause only small oscillations of the contact spin density value (see Figure 1b).

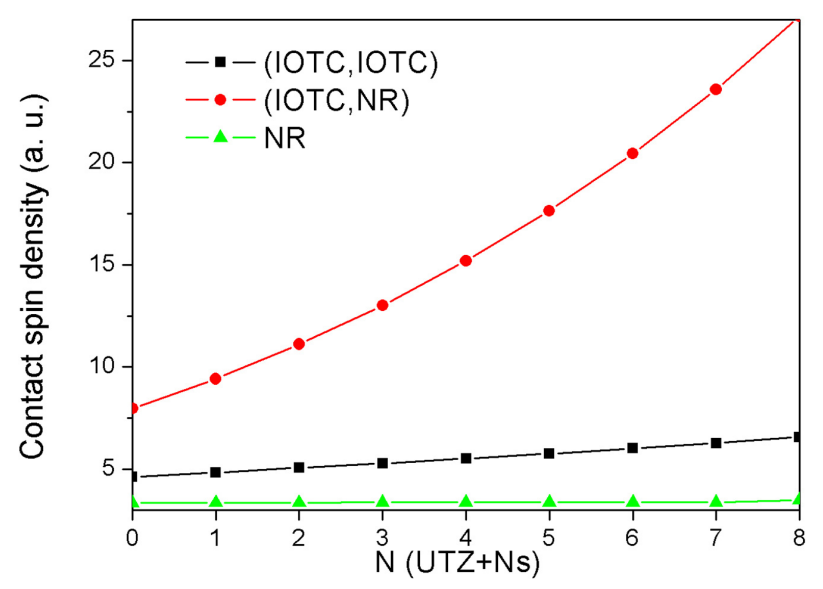

Fig. 1a. The dependence of IOTC contact spin density at the $\mathrm{PCH}$ nucleus of $\mathrm{Cu}$ on the number of additional tight $s$ Gaussians within the $\mathrm{UTZ}+\mathrm{Ns}$ basis set.
This has been found also for the contact electron densities of radon [Bučinský et al. 2011] and iron atoms [Mastalerz et al. 2010]. Such saturation of the value of the contact density is closely related to a situation when the exponent values of the tight $s$ Gaussians reach the exponent of the Gaussian nucleus [Knecht, Saue, van Wüllen, personal communications].

The influence of the FN model as well as the inclusion of tight $s$ Gaussians on the NR contact spin/ electron densities is negligible (impact is less than $1 \%$ ), see Figures 1(a), 1(b) and Tables 1, 2. The influence of additional tight $p$ Gaussians on the contact spin density of copper was found negligible and tight $d$ Gaussians have no influence on the contact spin density at all, see Table 3 . Nonetheless, the additional tight $p$ Gaussians have been found important in the case of relativistic calculations of hyperfine coupling constants [Malkin et al. 2004, Malkin et al. 2006]. In addition, the impact of tight $p$ Gaussians is found more pronounced in the contact electron densities [Mastalerz et al. 2010] when using contracted basis sets. Last but not least, the considered electron configuration of copper is of pure $s$ character, what can also cause a smaller contribution of higher angular momentum tight Gaussians to the contact densities.

\section{Relativistic effects and PCE}

Obviously, PGE cannot be neglected in the IOTC/ DKH2 calculations of contact spin/electron density of $\mathrm{Cu}$, see Tables 1 and 2. The PCE contaminated (IOTC $_{\mathrm{PCE}}$ and DKH $2_{\mathrm{PCE}}$ ) contact electron/spin densities are overestimated in comparison with IOTC and DKH2 values by a factor of roughly two in the case of the FN nucleus model (for both

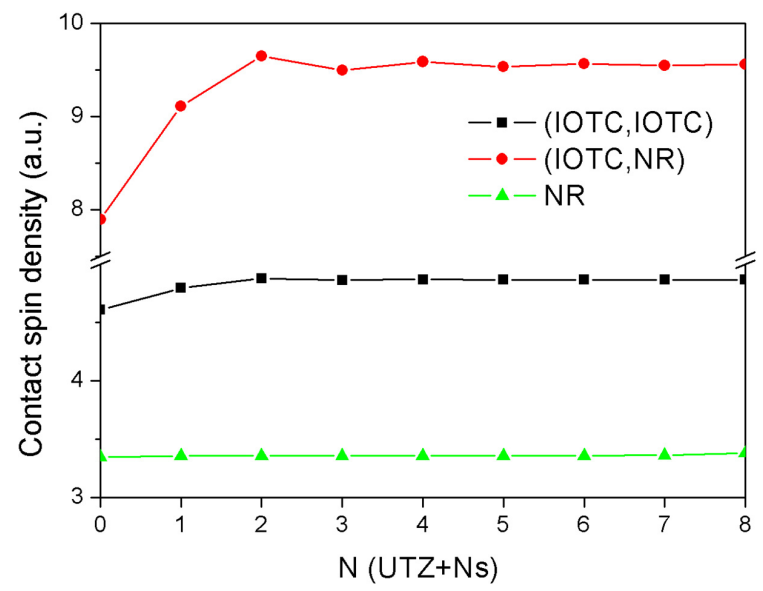

Fig. 1b. The dependence of IOTC contact spin density at the FN nucleus of $\mathrm{Cu}$ on the number of additional tight $s$ Gaussians within the $\mathrm{UTZ}+\mathrm{Ns}$ basis set. 
Tab. 1. Scalar NR, IOTC and DKH2 contact spin densities of $\mathrm{Cu}, \mathrm{Ag}, \mathrm{Au}$ (in a.u.) at UHF level of theory. The impact of relativistic effects including PCE, nucleus model and the number of tight $s$ Gaussian functions.

\begin{tabular}{|c|c|c|c|c|c|c|c|}
\hline $\mathbf{C u}$ & & & & & & & \\
\hline & NR & IOTC $_{\mathrm{PCE}}$ & IOTC & $\mathrm{DKH} 2_{\mathrm{PCE}}$ & $\mathrm{DKH}_{2}$ & DKH2 $2_{1}$ & DKH2 \\
\hline $\mathrm{UTZ}^{\mathrm{a}}$ & $3.3460^{\mathrm{d}}$ & $7.9111^{\mathrm{d}}$ & $4.6114^{\mathrm{d}}$ & 7.8931 & 6.2112 & 4.3096 & 4.6585 \\
\hline $\mathrm{UTZ}+10 \mathrm{~s}^{\mathrm{a}}$ & $3.3581^{\mathrm{c}}$ & 9.5113 & 4.8691 & 9.5113 & 7.2205 & 4.2785 & 4.9934 \\
\hline $\mathrm{UTZ}^{\mathrm{b}}$ & $3.3482^{\mathrm{d}}$ & $7.9568^{\mathrm{d}}$ & $4.6193^{\mathrm{d}}$ & 7.9381 & 6.2400 & 4.3117 & 4.6674 \\
\hline $\mathrm{UTZ}+10 \mathrm{~s}^{\mathrm{b}}$ & $3.3717^{c}$ & 36.0282 & 7.3366 & 33.4004 & 21.1026 & -1.4652 & 11.0473 \\
\hline \multicolumn{8}{|l|}{ Ag } \\
\hline & NR & IOTC $_{\mathrm{PCE}}$ & IOTC & $\mathrm{DKH} 2_{\mathrm{PCE}}$ & $\mathrm{DKH}_{0}$ & $\mathrm{DKH} 2_{1}$ & DKH2 \\
\hline $\mathrm{UTZ}^{\mathrm{a}}$ & $5.4138^{\mathrm{d}}$ & $34.2910^{\mathrm{d}}$ & $13.7498^{\mathrm{d}}$ & 33.8305 & 22.9164 & 10.4577 & 14.7594 \\
\hline $\mathrm{UTZ}+6 \mathrm{~s}^{\mathrm{a}}$ & 5.4135 & 33.8880 & 13.6972 & 33.4427 & 22.6903 & 10.4965 & 14.6738 \\
\hline \multicolumn{8}{|l|}{$\mathrm{Au}$} \\
\hline & NR & $\mathrm{IOTC}_{\mathrm{PCE}}$ & IOTG & $\mathrm{DKH} 2_{\mathrm{PCE}}$ & $\mathrm{DKH}_{2}$ & $\mathrm{DKH}_{2}$ & DKH2 \\
\hline $\mathrm{UTZ}^{\mathrm{a}}$ & $9.8166^{\mathrm{d}}$ & $362.6563^{\mathrm{d}}$ & $119.8741^{\mathrm{d}}$ & 350.3748 & 207.0734 & 79.8522 & 136.1980 \\
\hline $\mathrm{UTZ}+4 \mathrm{~s}^{\mathrm{a}}$ & 9.8182 & 362.7617 & 119.8860 & 350.4256 & 207.0787 & 79.8508 & 136.2009 \\
\hline
\end{tabular}

${ }^{\mathrm{a}}$ Finite nucleus $(\mathrm{FN})$ model

${ }^{\mathrm{b}}$ Point charge $(\mathrm{PCH})$ nucleus model

${ }^{c}$ Results obtained for the UTZ+6 $s$ basis set

${ }^{\mathrm{d}}$ Ref. [Malček et al. 2013]

Tab. 2. Scalar NR, IOTC and DKH2 contact electron densities of Cu atom (in a.u.) at UHF level of theory. The impact of relativistic effects including PCE, nucleus model and the number of tight $s$ Gaussian functions

\begin{tabular}{|c|c|c|c|c|c|c|c|}
\hline \multicolumn{8}{|l|}{$\mathbf{C u}$} \\
\hline & NR & IOTC $_{\mathrm{PCE}}$ & IOTC & $\mathrm{DKH} 2_{\mathrm{PCE}}$ & $\mathrm{DKH}_{2}$ & $\mathrm{DKH}_{1}$ & DKH2 \\
\hline $\mathrm{UTZ}^{\mathrm{a}}$ & $16497.76^{\mathrm{d}}$ & $35862.20^{\mathrm{d}}$ & $20996.62^{\mathrm{d}}$ & 35780.84 & 28309.57 & 19612.28 & 21214.46 \\
\hline $\mathrm{UTZ}+10 \mathrm{~s}^{\mathrm{a}}$ & $16558.70^{c}$ & 43132.44 & 22167.47 & 43132.44 & 32904.14 & 19464.50 & 22738.61 \\
\hline $\mathrm{UTZ}^{\mathrm{b}}$ & $16508.27^{\mathrm{d}}$ & $36069.64^{\mathrm{d}}$ & $21032.37^{\mathrm{d}}$ & 35985.06 & 28440.54 & 19621.50 & 21254.46 \\
\hline $\mathrm{UTZ}+10 \mathrm{~s}^{\mathrm{b}}$ & $16624.10^{c}$ & 162171.32 & 32579.81 & 150256.64 & 95278.65 & -7777.43 & 49621.44 \\
\hline
\end{tabular}

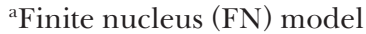

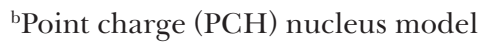

${ }^{\mathrm{c}}$ Results obtained for the $\mathrm{UTZ}+6 s$ basis set

${ }^{\mathrm{d}}$ Ref. [Malček et al. 2013]

$\mathrm{UTZ}$ and $\mathrm{UTZ}+10$ s basis sets) and in the case of the PCH model when using the UTZ basis set. PCE in the PCH UTZ+10s IOTC/DKH2 calculations of contact spin densities is even more dramatic (more than a factor of three).

PCE corrected IOTC and DKH2 contact spin densities at the FN model are in a good agreement with each other (the difference is around $3 \%$, relative to the IOTC value). In the case of $\mathrm{PCH}$ nucleus model in the connection with the $\mathrm{UTZ}+10 s$ basis set, this difference is considerably larger (around $50 \%$ ), see Table 1 . The $\mathrm{DKH} 2_{1}$ contact densities are in a better agreement with the DKH2 level of theory than the DKH $2_{0}$ one in the case of the FN model. Similar results are found also for the $\mathrm{PCH}$ nucleus model if the tight $s$ Gaussians are not included (a large basis set artifact). In the case of the highly augmented $(\mathrm{UTZ}+10 s)$ basis set, the PCH DKH2 1 contact spin/ electron density of $\mathrm{Cu}$ is negative (see Tables 1, 2). This is completely in agreement with the study [Bučinský et al. 2011], where this phenomenon was discussed in more detail. Particularly, some of the terms created in the DK 1 transformation within the PCE correction of the total electron density at the $\mathrm{PCH}$ nucleus are large in value and negative, and need to be counter-balanced by the terms from the DK2 transformation [Wolf and Reiher 2006a, Wolf and Reiher 2006b, Bučinský et al. 2010, Bučinský et al. 2011] if more tight $s$ Gaussians are included in the basis set. This shows on the failure of the 
Tab. 3. The impact of tight $s, p$ and $d$ Gaussian functions on the value of contact spin density of $\mathrm{Cu}$ atom at the IOTC/FN level of theory for both $\mathrm{FN}$ and $\mathrm{PCH}$ nucleus model

\begin{tabular}{lll}
\hline Gu & & \\
\hline Basis set & FN & PCH \\
\hline UTZ & $4.6114^{\mathrm{a}}$ & $4.6193^{\mathrm{a}}$ \\
$\mathrm{UTZ}+1 \mathrm{~s}$ & 4.7960 & 4.8365 \\
$\mathrm{UTZ}+2 \mathrm{~s}$ & $4.8783^{\mathrm{a}}$ & $5.0646^{\mathrm{a}}$ \\
$\mathrm{UTZ}+2 \mathrm{~s} 1 \mathrm{p}$ & 4.8783 & 5.0646 \\
$\mathrm{UTZ}+2 \mathrm{~s} 2 \mathrm{p}$ & 4.8782 & 5.0646 \\
$\mathrm{UTZ}+3 \mathrm{~s}$ & 4.8651 & 5.2839 \\
$\mathrm{UTZ}+4 \mathrm{~s}$ & $4.8707^{\mathrm{a}}$ & $5.5246^{\mathrm{a}}$ \\
$\mathrm{UTZ}+4 \mathrm{~s} 2 \mathrm{p}$ & 4.8706 & 5.5245 \\
$\mathrm{UTZ}+4 \mathrm{~s} 2 \mathrm{p} 1 \mathrm{~d}$ & 4.8705 & 5.5244 \\
$\mathrm{UTZ}+4 \mathrm{~s} 2 \mathrm{p} 2 \mathrm{~d}$ & 4.8705 & 5.5244 \\
$\mathrm{UTZ}+5 \mathrm{~s}$ & 4.8683 & 5.7594 \\
$\mathrm{UTZ}+6 \mathrm{~s}$ & 4.8693 & 6.0220 \\
\hline
\end{tabular}

${ }^{a}$ Ref. [Malček et al. 2013]
DK1 PCE correction for the DKH2 wave function and the need to account for the DK2 transformation. The found trends of the contact spin density, in regards of relativistic effects, PCE, nucleus size and basis set quality at the nucleus are similar to the ones found for the electron density [Mastalerz et al. 2010, Knecht et al. 2011, Bučinský et al. 2011, Bučinský et al. 2012].

An interesting point is also the contribution of individual orbitals to the contact UHF spin density. Of course, the unpaired electron in the $4 \mathrm{~s}$ shell has the dominant contribution, see Table 4 . For comparison, the contact spin density of the DCH $4 \mathrm{~s}$ spinorbitals is also shown in Table 4 . Nonetheless, remaining $s$ orbitals have non-negligible contributions to the UHF contact spin density as well (spin polarization), all together about $11.3 \%$. The contributions from scalar $p$ orbitals are negligible (see Table 4), although they have a non-zero contact electron density [Mastalerz et al. 2010, Bučinský et al. 2011], see Table 4. It is worth to point out that the PCE corrected scalar $p$ orbitals

Tab. 4. The orbital contributions to the value of contact spin densities of $\mathrm{Cu}, \mathrm{Ag}, \mathrm{Au}$ atoms at the IOTC/ FN level of theory, using UTZ+10s basis sets $\left(\rho_{\alpha, \mathrm{i}}\right.$ is $\alpha$-electron density and $\rho_{\mathrm{s}, \mathrm{i}}$ is spin density).

\begin{tabular}{|c|c|c|c|c|c|c|}
\hline \multirow[b]{2}{*}{$\mathrm{i}$} & \multicolumn{2}{|c|}{$\mathbf{C u}$} & \multicolumn{2}{|c|}{ Ag } & \multicolumn{2}{|c|}{$\mathbf{A u}$} \\
\hline & $\rho_{\alpha, \mathrm{i}}$ & $\rho_{\mathrm{s}, \mathrm{i}}$ & $\rho_{\alpha, i}$ & $\rho_{\mathrm{s}, \mathrm{i}}$ & $\rho_{\alpha, \mathrm{i}}$ & $\rho_{\mathrm{s}, \mathrm{i}}$ \\
\hline $1 \mathrm{~s}$ & 9959.7898 & 0.1260 & 63936.2298 & 0.2281 & 900425.8618 & 1.4734 \\
\hline $2 \mathrm{~s}$ & 978.6343 & 0.2515 & 7343.6122 & 0.2872 & 134915.9235 & 1.2831 \\
\hline \multirow[t]{3}{*}{$2 p$} & 1.6054 & 0.0002 & 28.2004 & 0.0004 & 814.3607 & 0.0021 \\
\hline & 1.6054 & 0.0002 & 28.2004 & 0.0004 & 814.3607 & 0.0021 \\
\hline & 1.6054 & 0.0002 & 28.2004 & 0.0004 & 814.3607 & 0.0021 \\
\hline $3 \mathrm{~s}$ & 137.9501 & 0.1723 & 1390.8536 & 0.3086 & 30922.5224 & 1.3778 \\
\hline \multirow[t]{3}{*}{$3 p$} & 0.2187 & -0.0006 & 5.7680 & -0.0002 & 214.4885 & 0.0003 \\
\hline & 0.2187 & -0.0006 & 5.7680 & -0.0002 & 214.4885 & 0.0003 \\
\hline & 0.2187 & -0.0006 & 5.7680 & -0.0002 & 214.4885 & 0.0003 \\
\hline $4 \mathrm{~s}$ & 4.3204 & 4.3204 & 242.9496 & 0.7559 & 7714.2856 & 1.8349 \\
\hline \multirow[t]{3}{*}{$4 p$} & & & 0.8957 & -0.0024 & 52.7849 & -0.0067 \\
\hline & & & 0.8957 & -0.0024 & 52.7849 & -0.0067 \\
\hline & & & 0.8957 & -0.0024 & 52.7849 & -0.0067 \\
\hline $5 \mathrm{~s}$ & & & 12.1239 & 12.1239 & 1439.0052 & 7.1303 \\
\hline \multirow[t]{3}{*}{$5 p$} & & & & & 8.3296 & -0.0332 \\
\hline & & & & & 8.3296 & -0.0332 \\
\hline & & & & & 8.3296 & -0.0332 \\
\hline $6 s$ & & & & & 106.8887 & 106.8887 \\
\hline LL & & 4.8701 & & 13.7036 & & 119.9882 \\
\hline SS & & -0.0011 & & -0.0064 & & -0.1125 \\
\hline Total & & 4.8691 & & 13.6972 & & 119.8757 \\
\hline$n s(\mathrm{DCH})^{\mathrm{a}}$ & 4.3154 & & 12.1209 & & 108.3584 & \\
\hline$n s(\mathrm{DCH})^{\mathrm{b}}$ & 4.2869 & & 12.1801 & & 108.3649 & \\
\hline
\end{tabular}

${ }^{a}$ Valence $s$ orbital density from Grasp90 package [Dyall et al. 1989]

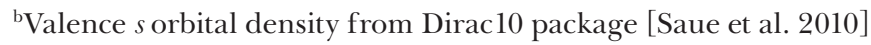


have an underestimated $s$ character in comparison with 2- or 4-component $p_{1 / 2}$ orbitals [Bučinský et al. 2011]. The small contribution of the $p$ orbitals can be further seen in the small (SS) component vs. large (LL) component contribution to the contact spin density, which can be obtained either by summing up separately the contributions of $s$ and $p$ orbitals separately.

\section{Ag and Au atoms}

The contact spin densities of $\mathrm{Ag}$ and $\mathrm{Au}$ atoms are shown in Tables 5 and 6, respectively, with emphasis on the relativistic effects (IOTC vs. NR), the quality of the basis set and the model of nucleus. The orbital contributions to the contact spin densities are shown in Table 4.

Size of the nucleus and the basis set quality at the nucleus The UTZ+N $s$ basis set was in the case of silver and gold atoms expanded by max. $6 s$ and/or $4 s$ Gaussian functions, respectively. Like in the case of $\mathrm{Cu}$, expanding the basis set with $p$ or $d$ functions has also a negligible effect on the contact spin/electron density. Contrary to $\mathrm{Cu}, \mathrm{FN}$ IOTC/DKH2 contact spin densities of $\mathrm{Ag}$ and $\mathrm{Au}$ are not affected by the additional tight $s$ Gaussians and are oscillatory for all $\mathrm{UTZ}+\mathrm{N} s$ basis sets (see Tables 1, 5 and 6). This is caused by the fact, that the largest exponents in the original UTZ basis sets of Ag and Au $\left(2.5914 \times 10^{+08}\right.$ and $5.1452 \times 10^{+10}$, respectively $)$ are already larger than the exponents of the Gaussian nucleus $\left(2.0389 \times 10^{+08}\right.$ and $\left.1.4223 \times 10^{+08}\right)$ [Malček et al. 2013].

Differences between the IOTC contact spin densities obtained for the FN and PCH nucleus models are mostly pronounced for the Au atom (see Table $6)$. The PCH IOTC contact spin density is approx. 2.3-times bigger than the FN IOTC one in the case of $\mathrm{Ag}(\mathrm{UTZ}+6 s)$. In the case of $\mathrm{Au}(\mathrm{UTZ}+4 s)$ this factor (PCH vs. FN) is nearly 18. This is completely in agreement with the fact that with the growing atomic number the inclusion of the $\mathrm{FN}$ model becomes more important in regard of the singular behavior of the relativistic densities at the PCH nucleus model. This obviously holds also for the spin and not only for the electron density, hand in hand with the $s$ electron configuration of the studied atoms. NR spin/electron densities of $\mathrm{Ag}$ and $\mathrm{Au}$ atoms are by less than $1 \%$ affected by the effect of the size of nucleus.

Tab. 5. Scalar NR and IOTC contact spin densities of Ag atom (in a.u.) at UHF level of theory. The impact of relativistic effects including PCE, nucleus model and the number of tight $s$ Gaussian functions.

\begin{tabular}{lcccccc}
\hline Rel. approach & IOTC & \multicolumn{3}{c}{ IOTC } \\
Nucleus model & FN & PCH & FN & PCH & FN & PCH \\
\hline UTZ & $13.7498^{\mathrm{a}}$ & $15.5432^{\mathrm{a}}$ & $34.2910^{\mathrm{a}}$ & $45.7752^{\mathrm{a}}$ & $5.4138^{\mathrm{a}}$ & $5.4456^{\mathrm{a}}$ \\
$\mathrm{UTZ}+1 \mathrm{~s}$ & 13.6692 & 17.3913 & 33.5686 & 58.8208 & 5.4135 & 5.4470 \\
$\mathrm{UTZ}+2 \mathrm{~s}$ & $13.7037^{\mathrm{a}}$ & $19.5250^{\mathrm{a}}$ & $34.0210^{\mathrm{a}}$ & $75.4426^{\mathrm{a}}$ & 5.4137 & 5.4498 \\
$\mathrm{UTZ}+3 \mathrm{~s}$ & 13.6883 & 21.8333 & 33.7380 & 95.8020 & 5.4136 & 5.4502 \\
$\mathrm{UTZ}+4 \mathrm{~s}$ & $13.6952^{\mathrm{a}}$ & $24.5031^{\mathrm{a}}$ & $33.9104^{\mathrm{a}}$ & $121.3030^{\mathrm{a}}$ & 5.4134 & 5.4513 \\
$\mathrm{UTZ}+5 \mathrm{~s}$ & 13.6921 & 27.5890 & 33.8070 & 153.7370 & 5.4140 & 5.4516 \\
$\mathrm{UTZ}+6 \mathrm{~s}$ & 13.6936 & 30.7447 & 33.8683 & 191.6559 & 5.4135 & 5.4521 \\
\hline
\end{tabular}

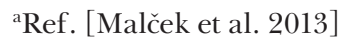

Tab. 6. Scalar NR and IOTC contact spin densities of Au atom (in a.u.) at UHF level of theory. The impact of relativistic effects including PCE, nucleus model and the number of tight $s$ Gaussian functions.

\begin{tabular}{lcccccc}
\hline Rel. approach & IOTC & \multicolumn{3}{c}{ IOTC $_{\text {PCE }}$} & NR \\
Nucleus model & FN & PCH & FN & PCH & FN & PCH \\
\hline UTZ & $119.8741^{\mathrm{a}}$ & $500.4707^{\mathrm{a}}$ & $362.6565^{\mathrm{a}}$ & $2728.8416^{\mathrm{a}}$ & $9.8166^{\mathrm{a}}$ & $9.9694^{\mathrm{a}}$ \\
UTZ+1s & 119.8881 & 715.4043 & 362.8430 & 4410.1196 & 9.8181 & 9.9693 \\
UTZ+2s & $119.8823^{\mathrm{a}}$ & $1032.3769^{\mathrm{a}}$ & $362.7231^{\mathrm{a}}$ & $7151.5267^{\mathrm{a}}$ & 9.8176 & 9.9695 \\
UTZ+3s & 119.8844 & 1478.0086 & 362.7952 & 11500.1425 & 9.8175 & 9.9695 \\
UTZ+4s & $119.8860^{\mathrm{a}}$ & $2134.7824^{\mathrm{a}}$ & $362.7617^{\mathrm{a}}$ & $18521.6598^{\mathrm{a}}$ & 9.8182 & 9.9700 \\
\hline
\end{tabular}

${ }^{a}$ Ref. [Malček et al. 2013] 


\section{Relativistic effects and PCE}

Differences between NR and IOTC contact spin densities are, as expected, far larger in the case of $\mathrm{Au}$ than in the cases of Ag or $\mathrm{Cu}$. The NR contact spin density of $\mathrm{Ag}$ accounts for about $40 \%$, while for $\mathrm{Au}$ it is only around $8 \%$, of the value obtained at the IOTC level of theory. The PCE contaminated IOTC $_{\mathrm{PCE}}$ contact spin density at $\mathrm{FN}$ is about three times larger than the PCE corrected one for both the Ag and $\mathrm{Au}$ atoms, see Tables 5 and 6, respectively. In the case of PCH nucleus model, PCE is much more pronounced because of the "singular behavior" of the contact spin density (similar holds also for the electron density, not shown).

In the case of DKH2 calculations of contact spin density the trends of PCE and the order of the PCE correction agree well with those found for the copper atom, see Table 1. This means that in the case of the FN model, the DKH2 contact spin density is in good agreement with the more rigorous IOTC value. The $\mathrm{DKH}_{2}$ contact spin density is underestimated and $\mathrm{DKH}_{2}$ overestimated in comparison with the IOTC level of theory. The PCE contaminated $\mathrm{DKH} 2_{\mathrm{PCE}}$ and IOTC $_{\mathrm{PCE}}$ contact spin densities are close each other and $\mathrm{DKH} 2_{\mathrm{PCE}}$ is slightly smaller than IOTC $_{\mathrm{PCE}}$ contact spin density. Herein, only the results for the $\mathrm{FN}$ model have been considered.

The orbital contributions to the IOTC FN contact spin densities of $\mathrm{Ag}$ and $\mathrm{Au}$ are shown in Table 4. As it holds for the $\mathrm{Cu}$ atom, the dominant contribution to the contact spin density comes from the unpaired valence $s$ electron. The remaining $s$ orbitals contribute again by about $11.5 \%$ and $10.8 \%$ (Ag and $\mathrm{Au}$, respectively) to the final value of the spin density. The contribution of PCE corrected $p$ orbitals (small component) to the contact spin density is even in the case of Au less than $0.1 \%$. The $5 s$ and $6 s$ IOTC contact orbital densities of $\mathrm{Ag}$ and $\mathrm{Au}$, respectively, agree well with the DCH values, see Table 4. Small differences are caused mostly by the missing two electron terms of DCH within the IOTC transformation [Seino and Hada 2008, Seino et al. 2010].

\section{Conclusions}

The key factors in the calculations of contact spin densities of the coinage metal $(\mathrm{Cu}, \mathrm{Ag}, \mathrm{Au})$ atoms are the inclusion of relativistic effects hand in hand with the inclusion of finite model of nucleus. In addition, in the case of quasirelativistic calculations like DKH2 and/or IOTC the treatment of PCE shall not be forgotten. In accordance with the previous paper [Bučinský et al. 2011], the PCH DKH2 $2_{1}$ contact electron/spin densities are "ill behaved" (negative) if tight enough $s$ Gaussians $(\mathrm{N})$ are included in the $\mathrm{UTZ}+\mathrm{Ns}$ basis set.
Furthermore, the expansion of the basis set by tight $s$ Gaussians in the FN DKH2/IOTC calculations is of importance in the case of the $\mathrm{Cu}$ atom. At least two tight $s$ Gaussians are required to achieve reasonable convergence of contact spin density of $\mathrm{Cu}$. Because the original UTZ basis sets for Ag and Au [Balabanov and Petersen 2005, Petersen and Puzzarini 2005] contain larger $s$ Gaussian exponents than the exponents in the Gaussian nucleus (FN) model [Visscher and Dyall 1997], the additional tight $s$ Gaussians have not a dramatic effect on their contact spin/electron density values. In the case of PCH nucleus model the analytic/numeric contact spin density at the relativistic level of theory is singular at the nucleus, i.e. contact spin density is nearly a linear function of the number of extra tight $s$ Gaussian functions added to the basis set. Additional $p$ or $d$ Gaussians have a negligible influence on the contact spin densities of the studied atoms.

\section{Acknowledgement}

The financial support was obtained from APVV (Contract No. APVV-0202-10) and VEGA (Contract No. 1/0327/12). We are grateful to the HPC Center at the Slovak University of Technology in Bratislava, which is a part of the Slovak Infrastructure of High Performance Computing (SIVVP project, ITMS code26230120002, funded by the European Regional Development Funds), for the computational time and resources made available.

\section{References}

Balabanov NB, Peterson KA (2005) J. Chem. Phys. 123: 064107(15).

Barysz M, Mentel Ł, Leszczyński J (2009) J. Chem. Phys. 130: 164114(8).

Barysz M, Sadlej AJ, Snijders JG (1997) Int. J. Quantum Chem. 65: 225-239.

Barysz M, Sadlej AJ (2001) J. Mol. Struct. 573: 181-200.

Barysz M, Sadlej AJ (2002) J. Chem. Phys. 116: 26962704.

Bučinský L, Biskupič S, Jayatilaka D (2010) J. Chem. Phys. 133: 174125(14).

Bučinský L, Biskupič S, Jayatilaka D (2011) Theor. Chem. Acc. 129: 181-197.

Bučinský L, Biskupič S, Jayatilaka D (2012) Chem. Phys. 395: 44-53.

Douglas M, Kroll NM (1974) Ann. Phys. 82: 89-155.

Dyall KG, Faegri K (2007) Introduction to Relativistic Quantum Chemistry, Oxford University Press, New York.

Dyall KG, Grant IP, Johnson CT, Parpia FA, Plummer EP (1989) Grasp, Comp. Phys. Commun. 55: 425-456.

Foldy LL, Wouthuysen SA (1950) Phys. Rev. 78: 29-36.

Hess BA (1985) Phys. Rev. A 32: 756-763.

Iliaš M, Kellö V, Urban M (2010) Acta Physica Slovaca 30: 259-391.

Iliaš M, Saue T (2007) J. Chem. Phys. 126: 064102(9). 
Jayatilaka D, Grimwood DJ (2000) Tonto: A Research Tool for Quantum Chemistry, The University of Western Australia, Nedlands, Western Australia, Australia, http://www.theochem.uwa.edu.au

Kedziera D, Barysz M (2007) Chem. Phys. Lett. 446: 176-181.

Knecht S, Fux S, van Merr R, Visscher L, Reiher M, Saue T (2011) Theor. Chem. Acc. 129: 631-650.

Knecht S, personal communications.

Kutzelnigg W (2004) Chapter 5. Fundamentals of Nonrelativistic and Relativistic Theory of NMR and EPR Parameters in Calculations of NMR and EPR Parameters, WILEY-VCH, Weinheim.

Malček M, Bučinský L, Biskupič S, Jayatilaka D (2013) Chem. Phys. Lett. 580: 152-159.

Malkin E, Malkin I, Malkina OL, Malkin VG, Kaupp M (2006) Phys. Chem. Chem. Phys. 8: 4079-4085.

Malkin I, Malkina OL, Malkin VG, Kaupp M (2004) Chem Phys. Lett. 396: 268-276.

Mastalerz R, Lindh R, Reiher M (2008) Chem. Phys. Lett. 465: 157-164.

Mastalerz R, Widmark PO, Roos BO, Lindh R, Reiher M (2010) J. Chem. Phys. 133: 144111(10).

Nakajima T, Hirao K (2000) J. Chem. Phys. 113: 7786 7789.

Peng D, Reiher M (2012) Theor. Chem. Acc. 131: 1081(20).

Peterson KA, Puzzarini C (2005) Theor. Chem. Acc. 114: 283-296.
Pyykkö P (1988) Chem. Rev. 88: 563-594.

Reiher M, Wolf A (2004) J. Chem. Phys. 121: 20372047.

Reiher M, Wolf A (2004) J. Chem. Phys. 121: 1094510956.

Reiher M, Wolf A (2009) Relativistic Quantum Chemistry, WILEY-VCH, Weinheim.

Saue T, Visscher L, Jensen HJAa (2010) DIRAC, a relativistic ab initio electronic structure program, Release DIRAC10, http://dirac.chem.vu.nl

Saue T, personal communications.

Seino J, Hada M (2008) Chem. Phys. Lett. 461: 327-331.

Seino J, Uesegi W, Hada M (2010) J. Chem. Phys. 132: $164108(9)$.

Schwerdtfeger P (Ed.) (2002) Relativistic Electronic Structure Theory, Part 1. Fundamentals, Elsevier, Amsterdam.

Visscher L, Dyall KG (1997) Atom. Data Nucl. Data Tabl. 67: 207-224.

Wolf A, Reiher M, Hess BA (2002) J. Chem. Phys. 117: 9215-9226.

Wolf A, Reiher M (2006) J. Chem. Phys. 124: 064102(11).

Wolf A, Reiher M (2006) J. Chem. Phys. 124: 064103(10).

van Wüllen $\mathrm{C}$, personal communications. 